\title{
A Rare Case of Thyroid Metastasis Secondary to Hidden Undiagnosed Lung Adenocarcinoma
}

\author{
Marzouqi Salamaha, Mohannad Rajab ${ }^{\mathrm{b}, \mathrm{f}}$, Saif Alghamdic, \\ Abdullah Ashi ${ }^{\text {, }}$ Mohammed AlGarni ${ }^{\mathrm{e}}$
}

\begin{abstract}
The majority of thyroid lesions are primary in origin while secondary metastases to thyroid are considered a rare incidence. However, presentation of such cases with no signs of lung cancer can be extremely challenging to diagnose. Here, we present a 64-year-old man, an ex-smoker of 70 pack-years, who presented with a complaint of hoarseness of voice with associated dyspnea, choking episodes, weight loss, and hemoptysis. With no investigation abnormalities indicating lung adenocarcinoma, he was transferred to the Otolaryngology Department as being suspicious of thyroid cancer. A laryngoscopy demonstrated an immobile right vocal cord and pooling of secretions while a computed tomography (CT) scan showed a right thyroid lobe nodule, upper mediastinal lymphadenopathy, and pleural effusion. The patient underwent a total thyroidectomy. Biopsies from the lymph nodes and lung were obtained, and all demonstrated lung adenocarcinoma. Thus, a diagnosis of primary lung adenocarcinoma with thyroid and mediastinal lymph nodes metastases was established. Despite being a rare clinical presentation, thyroid metastasis should be considered and evaluated for a primary origin according to the associated clinical history and presentation.
\end{abstract}

Keywords: Thyroid; Metastasis; Lung; Adenocarcinoma

\section{Introduction}

Thyroid nodules are considered a common disease with a

Manuscript submitted July 29, 2020, accepted August 18, 2020

Published online August 28, 2020

aDepartment of Otolaryngology-Head and Neck Surgery, Ohud Hospital, AlMadinah Al-Munwarh, Saudi Arabia

${ }^{b}$ Department of Otolaryngology Head and Neck Surgery, Ministry of Health, Makkah, Saudi Arabia

'King Abdulaziz Medical City, National Guard Hospital, Jeddah, Saudi Arabia ${ }^{\mathrm{d} C o l l e g e ~ o f ~ M e d i c i n e-J e d d a h, ~ K i n g ~ S a u d ~ b i n ~ A b d u l a z i z ~ U n i v e r s i t y ~ f o r ~ H e a l t h ~}$ Sciences, King Abdulaziz Medical City, National Guard Health Affairs, Jeddah 21423, Saudi Arabia

'Otolaryngology-Head and Neck Surgery Section, King Saud Bin Abdulaziz University, Jeddah, Saudi Arabia

fCorresponding Author: Mohannad Rajab, Department of Otolaryngology Head and Neck Surgery, Ministry of Health, Makkah, Saudi Arabia.

Email:mohannad.k.rajab@gmail.com

doi: https://doi.org/10.14740/jmc3557 prevalence rate of $4-7 \%$ discoverable by palpation alone with a primary clinical significance towards the detection of malignancy [1]. It is estimated that $19-67 \%$ of thyroid nodules are only detectable when examined under ultrasound [2]. Cases of thyroid carcinoma with unknown origin have been described as occult cases especially in papillary thyroid carcinoma with no pathological findings to support a secondary diagnosis [3]. Even though in most cases of thyroid metastasis, the primary source is known, a significant rate of $20 \%$ has been reported to be occult [4]. Renal cell carcinoma is considered the most common primary site followed by lung, gastrointestinal, and breast cancer, respectively [4]. Furthermore, lung cancer incidence is rising with adenocarcinoma leading the race with $38.5 \%$ of all lung cancers followed by squamous cell carcinoma and large cell carcinoma [5, 6]. However, metastasis to thyroid secondary to lung lesion is considered an uncommon incident with few studies reporting it in the literature [7-9].

\section{Case Report}

A 64-year-old ex-smoker with type 2 diabetes (70 pack-years) man was transferred to the Otorhinolaryngology after being seen in the Pulmonology Department with a progressive gradual increase in hoarseness of voice for a duration of 6 months, shortness of breath exacerbated by exertion and when lying flat had choking attacks, which occurred with liquids only, weight loss and recent hemoptysis with no history of fever, dysphagia, pain, or tuberculosis exposure. Afterwards, pleural effusion drainage was done by the Pulmonology Department to relieve the symptoms and he was sent for cytology. Surprisingly, the lung computed tomography (CT) only showed a large left pleural effusion at the time with complete left lung collapse, no obstructive mass was present, and no signs of any lesion was reported (Fig. 1). Meanwhile, the pleural effusion cytology showed atypical cells, vacuolated cytoplasm, and prominent nucleoli in a background of macrophages, lymphocytes and blood with suspicion of malignancy. Six weeks later, another pleural tap for a recurrent pleural effusion was performed with the pleural fluid cytology being negative for tumor cells. After complete full body workup, the Pulmonary Department discovered a thyroid nodule and transferred the case to the Otorhinolaryngology with a provisional diagnosis of thyroid nodule due to the progressiveness of hoarseness of 


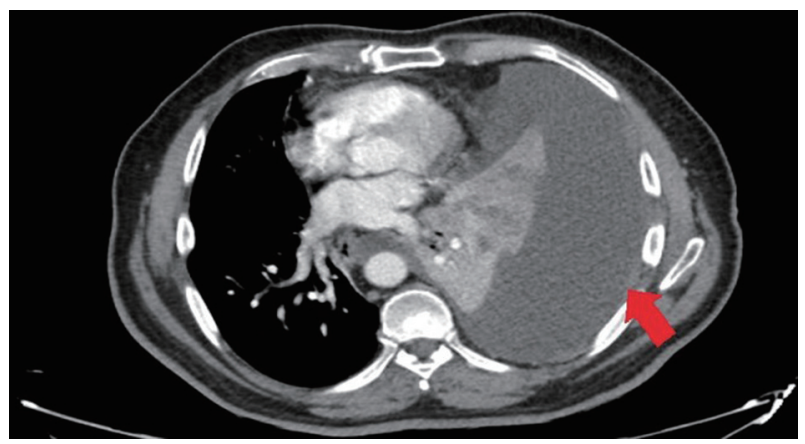

Figure 1. Lung CT showing a large left pleural effusion with complete left lung collapse with no obvious obstructive mass (arrow). CT: computed tomography.

\section{voice with lung metastasis.}

On head and neck examination, the tongue was deviated to the right with no other abnormalities found and no lymphadenopathy. A laryngoscopy showed a mobile left vocal cord and an immobile right vocal cord in the paramedian position with excessive pooling of hypopharyngeal secretions, most extensively in the right pyriform fossa. Further investigation of the thyroid showed a normal thyroid function tests (TFT) while a neck CT showed a right vocal cord paralysis with a right thyroid nodule about $1.5 \mathrm{~cm}$ (Fig. 2) and an upper mediastinal lymph node enlargement at the right tracheoesophageal groove that was causing the nerve irritation (Fig. 3 ). Fine needle aspiration (FNA) of the thyroid nodule was performed and was negative. The patient's hoarseness with the plural effusion was suspected to be related to a primary thyroid malignancy as primary source with possible lung metastasis.

Subsequently, it was decided to perform a right hemi-thyroidectomy with frozen section and a possible total thyroidectomy with neck dissection for further evaluation of the hoarseness and plural effusion cause. As a part of the pre-operative evaluation, the cardiothoracic team evaluated the patient and a CT chest was requested to be repeated. It demonstrated a left hilar mass with obstruction of the left upper lobe bronchus

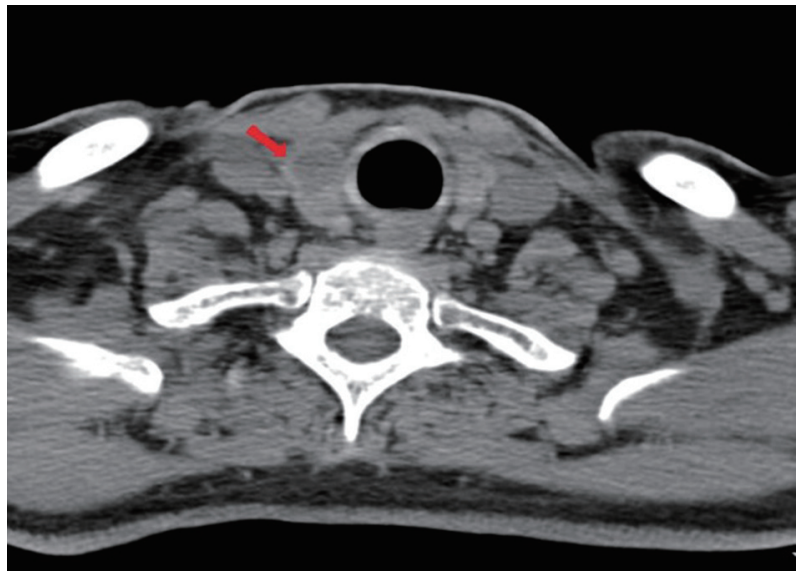

Figure 2. Neck CT showing a hypodense nodule within the right lobe of the thyroid gland (arrow). CT: computed tomography.

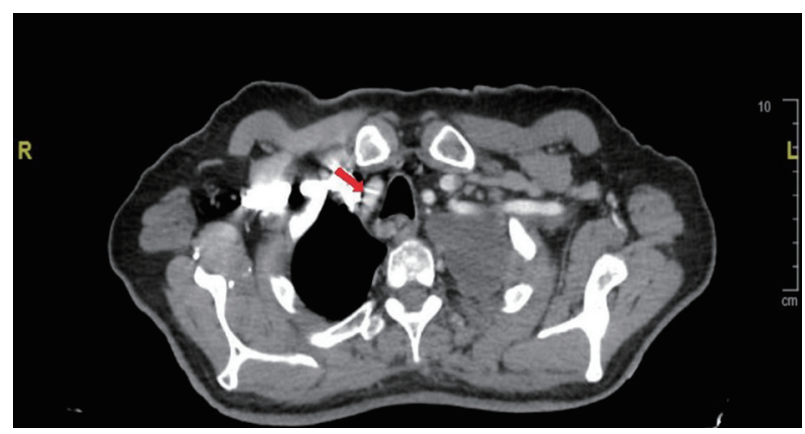

Figure 3. The mass effect at the right tracheoesophageal groove (arrow).

and lung collapse with two suspicious pulmonary nodules at the lateral segment of the left lower lobe with no other lesions seen at the time which were not visible in the previous CT (Fig. 1). According to the CT, the thoracic surgeon decided to do a flexible bronchoscopy with a possible biopsy from the left hilar mass concurrently with the right hemi-thyroidectomy. Even though a hemi-thyroidectomy was planned, the patient eventually underwent a total thyroidectomy with paratracheal lymph node biopsy without complications. The flexible bronchoscopy showed a completely obstructed left upper lobe by a mass with erythematous changes and white deposit on the entry. Multiple biopsies were taken, and bronchial washing was obtained and sent for cytology and histopathology. Post-operatively, he was started on levothyroxine $50 \mu \mathrm{g}$ with follow-up TFTs and a review of the pathology specimens at a later follow-up. His post-operative course was uneventful and he was discharged home without any complications. Later, the pathology report indicated the presence of lung adenocarcinoma from the left upper bronchus aspirate and metastatic lung adenocarcinoma was noted in the thyroid and lymph node specimens. The case was discussed by the tumor board for a further follow-up CT of chest, abdomen and pelvis to check for other metastatic lesions followed by cardiothoracic assessment of the lung adenocarcinoma. Unfortunately, the patient was not able to be followed up later by the cardiothoracic team as he had passed away.

\section{Discussion}

The majority of malignant thyroid nodules are primary malignancies with only $1.4-3 \%$ being due to metastasis to the thyroid [10]. Milovanovic et al described in the autopsies of 175 bodies the pattern of metastases of lung carcinoma that included liver, adrenal glands and bones as the most common form of metastasis from lung carcinoma while thyroid lesions were the least likely to occur with a rate of $1.7 \%$ [7]. Del Ciello et al reported that $90 \%$ of the overall lung carcinoma was missed on chest X-ray, 5\% were missed on CT scans and 5\% were missed during other investigational modalities [11]. Such errors were categorized as observer error, and mainly found in cases where only one clinician is responsible for reporting the lesion. The second cause is related to the lesion characteristics, which was the most probable in our case, as $29 \%$ of lung lesions with 
a diameter less than $10 \mathrm{~mm}$ were undetectable with different studies reporting even larger diameters being missed [11-13]. Lastly, the third reason for reporting errors according to Del Ciello et al is due to technicalities, whether it is picture quality or patient position [11]. Regardless, suspicion of thyroid malignancy is usually high with the presence of local symptoms, such as hoarseness of voice and choking spells, especially in cases of medullary carcinoma where the metastasis spreads to the lung as it is the third most common site of metastasis with $33 \%$ rate in such cases [14]. Therefore, inconclusive cytological pleural fluid analysis for such cases with no findings of a mass on the CT scan warrants further investigations. For example, Kaur et al reported that immunohistochemistry investigation of nuclear thyroid transcription factor-1 (TTF-1) with cytoplasmic positivity for cytokeratin-7 (CK7) can diagnose up to $71 \%$ of lung adenocarcinoma [15]. In another case of metastatic thyroid cancer from non-small cell lung carcinoma, an immunohistochemistry of the fluid from the FNA of the thyroid showed positive TFF-1 with negative thyroglobulin which supports the diagnosis of a primary lung carcinoma [8].

\section{Conclusions}

Thyroid metastatic lesions from lung adenocarcinoma are very rare and may occur in any smoker. Proper evaluation and investigation should be done thoroughly to pleural fluid and the thyroid aspirate as well as immunohistochemistry investigation to differentiate thyroid metastasis causes and not confuse it as a primary source of malignancy even in the cases where no presenting mass outside the site of the thyroid tumor is found.

\section{Acknowledgments}

None to declare.

\section{Financial Disclosure}

None to declare.

\section{Conflict of Interest}

None to declare.

\section{Informed Consent}

Informed consent was obtained from the patient's family to report the case.

\section{Author Contributions}

MS, MR, SA, AA and MA contributed to data collection, writing, proofreading and final editing of this report.

\section{Data Availability}

All the data supporting this report can be found in the text of the manuscript.

\section{References}

1. Popoveniuc G, Jonklaas J. Thyroid nodules. Med Clin North Am. 2012;96(2):329-349.

2. Tan GH, Gharib H. Thyroid incidentalomas: management approaches to nonpalpable nodules discovered incidentally on thyroid imaging. Ann Intern Med. 1997;126(3):226-231.

3. Xu B, Scognamiglio T, Cohen PR, Prasad ML, Hasanovic A, Tuttle RM, Katabi N, et al. Metastatic thyroid carcinoma without identifiable primary tumor within the thyroid gland: a retrospective study of a rare phenomenon. Hum Pathol. 2017;65:133-139.

4. Nixon IJ, Coca-Pelaz A, Kaleva AI, Triantafyllou A, Angelos P, Owen RP, Rinaldo A, et al. Metastasis to the thyroid gland: a critical review. Ann Surg Oncol. 2017;24(6):1533-1539.

5. Barta JA, Powell CA, Wisnivesky JP. Global epidemiology of lung cancer. Ann Glob Health. 2019;85(1):8.

6. Dela Cruz CS, Tanoue LT, Matthay RA. Lung cancer: epidemiology, etiology, and prevention. Clin Chest Med. 2011;32(4):605-644.

7. Milovanovic IS, Stjepanovic M, Mitrovic D. Distribution patterns of the metastases of the lung carcinoma in relation to histological type of the primary tumor: An autopsy study. Ann Thorac Med. 2017;12(3):191-198.

8. Namad T, Wang J, Shipley R, Abdel Karim N. Thyroid metastasis from nonsmall cell lung cancer. Case Rep Oncol Med. 2013;2013:208213.

9. Dao A, Jabir H, Taleb A, Benchakroun N, Bouchbika Z, Nezha T, Jouhadi H, et al. Lung adenocarcinoma with thyroid metastasis: a case report. BMC Res Notes. 2017;10(1):130.

10. Nakhjavani MK, Gharib H, Goellner JR, van Heerden JA. Metastasis to the thyroid gland. A report of 43 cases. Cancer. 1997;79(3):574-578.

11. Del Ciello A, Franchi P, Contegiacomo A, Cicchetti G, Bonomo L, Larici AR. Missed lung cancer: when, where, and why? Diagn Interv Radiol. 2017;23(2):118-126.

12. Monnier-Cholley L, Arrive L, Porcel A, Shehata K, Dahan H, Urban T, Febvre M, et al. Characteristics of missed lung cancer on chest radiographs: a French experience. Eur Radiol. 2001;11(4):597-605.

13. Wu MH, Gotway MB, Lee TJ, Chern MS, Cheng HC, Ko JS, Sheu MH, et al. Features of non-small cell lung carcinomas overlooked at digital chest radiography. Clin Radiol. 2008;63(5):518-528.

14. Smit J. Treatment of advanced medullary thyroid cancer. Thyroid Res. 2013;6(Suppl 1):S7.

15. Kaur G, Nijhawan R, Gupta N, Singh N, Rajwanshi A. Pleural fluid cytology samples in cases of suspected lung cancer: An experience from a tertiary care centre. Diagn Cytopathol. 2017;45(3):195-201. 